Strongly damped quadratic matrix polynomials

\author{
Taslaman, Leo
}

2014

MIMS EPrint: 2014.10

Manchester Institute for Mathematical Sciences

School of Mathematics

The University of Manchester

\footnotetext{
Reports available from: http://eprints.maths.manchester.ac.uk/

And by contacting: The MIMS Secretary

School of Mathematics

The University of Manchester

Manchester, M13 9PL, UK
} 


\title{
STRONGLY DAMPED QUADRATIC MATRIX POLYNOMIALS *
}

\author{
LEO TASLAMAN $\dagger$
}

\begin{abstract}
We study the eigenvalues and eigenspaces of the quadratic matrix polynomial $M \lambda^{2}+s D \lambda+K$ as $s \rightarrow \infty$, where $M$ and $K$ are symmetric positive definite and $D$ is symmetric positive semi-definite. The work is motivated by its application to modal analysis of finite element models with strong linear damping. Our results yield a mathematical explanation of why too strong damping may lead to practically undamped modes such that all nodes in the model vibrate essentially in phase.
\end{abstract}

Key words. quadratic eigenvalue problem, principal angles, canonical angles, matrix polynomial, viscous damping, discrete damper, vibrating system

AMS subject classifications. 15A18, 15A22, 65F15, 70J10, 70J30, 70J50

1. Introduction. A way to prevent a structure from vibrating violently is to incorporate viscous dampers into the design. A viscous damper is a device that resists motion by producing a force proportional to the relative velocity of its ends raised to a power $\alpha$. In this paper we consider linear damping, which corresponds to dampers with $\alpha=1$. This value of $\alpha$ is the default for certain product lines of seismic dampers [1]. The resisting force produced by a viscous damper arises when fluid, trapped in a cylinder, is forced through small holes (see Figure 1). By adjusting the size of these holes, we can make the damper stronger. But stronger is not necessarily better: if a damper is too strong, it resembles a rigid component and hence has little purpose. This suggests that a structure with only very strong dampers should be quite similar to a structure without dampers. The goal of this paper is to investigate this phenomenon more rigorously for discretized structures. We will do this by studying the eigenvalues and eigenspaces of a related quadratic matrix polynomial.

Consider a finite element model of a structure with $r$ viscous dampers. If the model vibrates freely, the displacements of its nodes are given by the solutions to the equations of motion:

$$
M \ddot{u}(t)+s D \dot{u}(t)+K u(t)=0 .
$$

Here $M, s D$ and $K$ correspond to mass, the viscous dampers and stiffness, respectively. We assume these matrices are $n \times n$, real and symmetric positive semi-definite, and further that $M$ and $K$ are strictly positive definite. We also assume that each damper contributes to the damping matrix with a rank one term, so $\operatorname{rank}(D)=r$ and $D=R R^{T}$ for some real $n \times r$ matrix $R$. If $\|D\|=1$, say, the parameter $s$ determines the strength of the dampers, so larger $s$ corresponds to viscous dampers with smaller holes, and $s=0$ yields an undamped system.

We find the solutions to (1.1) by solving the quadratic eigenproblem

$$
P_{s}(\lambda) x=0, \quad s \geq 0,
$$

where $P_{s}(\lambda)=M \lambda^{2}+s D \lambda+K$. The spectrum of $P_{s}(\lambda)$ lies in the left half plane and is symmetric with respect to the real axis. Further, if $(-d+i \omega, x)$ is an eigenpair of

\footnotetext{
*Version of March 3, 2014. This work was supported by Engineering and Physical Sciences Research Council grant EP/I005293

†School of Mathematics, The University of Manchester, Manchester, M13 9PL, UK (leo.taslaman@manchester.co.uk)
} 


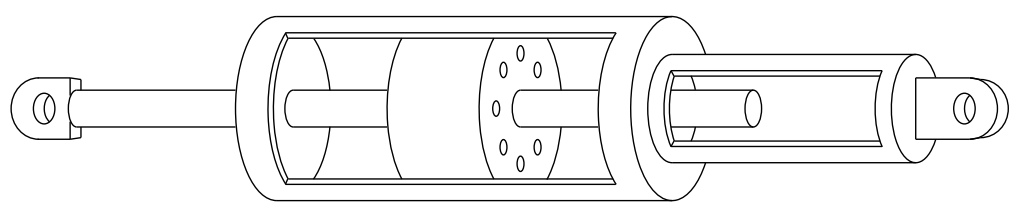

FIG. 1.1. A model of a viscous damper. The larger cylinder is filled with a fluid which is forced through holes in the piston head as the piston rod moves horizontally. This causes friction and energy is dissipated and released as heat.

$P_{s}(\lambda)$, where $d, \omega \in \mathbb{R}$, and $x$ is real if $\omega=0$, then

$$
u(t)=e^{-d t}(\cos (t \omega) \operatorname{Re}(x)-\sin (t \omega) \operatorname{Im}(x))
$$

is a real solution to (1.1) and is called a mode. ${ }^{1}$ We see that $d$ and $\omega$ correspond to damping and frequency, respectively. The solution (1.3) describes how the model switches between two configurations, given by $\operatorname{Re}(x)$ and $\operatorname{Im}(x)$, as it vibrates. If $x=v e^{i \theta}$ for some $v \in \mathbb{R}^{n}$ and $\theta \in \mathbb{R}$, then

$$
u(t)=e^{-d t}(\cos (t \omega) \cos (\theta)-\sin (t \omega) \sin (\theta)) v=e^{-d t} \cos (t \omega+\theta) v,
$$

and we see that these two configurations must coincide and that all nodes in the model vibrate in phase. Now, if $s=0$, it is well known that all eigenvalues are nonzero and purely imaginary, and that all eigenspaces have real bases and are pairwise $M$-orthogonal [8, Section 7.3]. In particular, all modes of an undamped model are undamped and those modes that correspond to simple eigenvalues are such that all nodes in the model vibrate in phase. We will see in Lemma 3.4 that this cannot be the case when damping is present.

To see the similarities between strongly damped structures and undamped ones, we will prove that the eigenvalues of $P_{s}(\lambda)$ approach nonzero points on the imaginary axis as $s \rightarrow \infty$, with the exception of $2 r$ real eigenvalues which correspond to overdamped modes (that is, non-oscillating modes). This implies that the considered model has $n-r$ practically undamped modes for large enough $s$. Regarding the eigenspaces of $P_{s}(\lambda)$ as $s \rightarrow \infty$, we will show the following. If two eigenvalues converge to distinct points on the imaginary axis, then the corresponding eigenspaces become more and more $M$-orthogonal in terms of the principal angles (defined in Section 2.1). Further, we will prove that the span of all eigenvectors associated with eigenvalues converging to a given point has an $M$-orthonormal basis that become more and more real in the sense that the norms of the imaginary parts go to zero. In particular, eigenvalues converging to points to which no other eigenvalues converge, are, for large enough $s$, associated with almost real eigenvectors. This corresponds to the case of simple eigenvalues for the undamped problem, and from (1.3) we see that the associated modes are such that all nodes in the model vibrate essentially in phase.

1.1. Outline. In Section 2 we introduce the notion of principal angles and establish two results, Proposition 2.4 and Proposition 2.5, which are needed for Section 4. In Section 3, we study the eigenvalues of $P_{s}(\lambda)$ as $s \rightarrow \infty$, and prove an eigenvalue location result which extends some early work by Lancaster [8]. In Section 4 we study the eigenspaces of $P_{s}(\lambda)$ as $s \rightarrow \infty$.

\footnotetext{
${ }^{1}$ The term 'mode' is ambiguous and is sometimes, although not in this paper, used to refer to an eigenvector.
} 
2. Preliminaries. In what follows, $\langle\cdot, \cdot\rangle$ denotes an arbitrary positive definite inner product on $\mathbb{C}^{n}$ and $\|\cdot\|$ denotes the induced norm. Further, for a subspace $\mathcal{X}$ we define $S(\mathcal{X})=\{x: x \in \mathcal{X},\|x\|=1\}$.

2.1. The principal angles. The angle between two nonzero vectors $u$ and $v$ is defined as

$$
\measuredangle(u, v)=\arccos \left(\frac{|\langle u, v\rangle|}{\|u\|\|v\|}\right) .
$$

To generalize the concept of angles to subspaces the principal angles (or canonical angles) are introduced. Given two $\operatorname{subspaces} \mathcal{U}$ and $\mathcal{V}$, such that $p=\operatorname{dim} \mathcal{U} \leq \operatorname{dim} \mathcal{V}=$ $q$, there are $p$ principal angles

$$
\theta_{1}(\mathcal{U}, \mathcal{V}) \leq \theta_{2}(\mathcal{U}, \mathcal{V}) \leq \cdots \leq \theta_{p}(\mathcal{U}, \mathcal{V})
$$

which all lie in $[0, \pi / 2]$. For convenience, we shall with $\theta_{\max }(\mathcal{U}, \mathcal{V})$ refer to $\theta_{p}(\mathcal{U}, \mathcal{V})$. The first principal angle is defined as

$$
\theta_{1}(\mathcal{U}, \mathcal{V})=\min \{\measuredangle(u, v): u \in S(\mathcal{U}), v \in S(\mathcal{V})\}=\measuredangle\left(u_{1}, v_{1}\right),
$$

where $u_{1}$ and $v_{1}$ are some minimizing vectors. The remaining angles are then defined recursively by

$$
\begin{aligned}
\theta_{i}(\mathcal{U}, \mathcal{V})= & \min \{\measuredangle(u, v): u \in S(\mathcal{U}), v \in S(\mathcal{V}), \\
& \left.\left\langle u, u_{j}\right\rangle=\left\langle v, v_{j}\right\rangle=0, j=1,2, \ldots, i-1\right\} \\
= & \measuredangle\left(u_{i}, v_{i}\right),
\end{aligned}
$$

where $u_{i}$ and $v_{i}$ are minimizing vectors. The vectors $u_{1}, u_{2}, \ldots, u_{p}$ and $v_{1}, v_{2}, \ldots, v_{p}$ are obviously not unique but the principal angles are. This is easily seen from the next theorem, which is due to Björck and Golub [2]. The proof in [2] is for the standard inner product, but it can easily be extended to an arbitrary inner product.

THEOREM 2.1. Suppose the inner product $\langle\cdot, \cdot\rangle$ corresponds to a symmetric positive definite matrix $A$, so $\langle x, y\rangle=x^{*} A y$ for any vectors $x$ and $y$. If the columns of $U$ and $V$ form $A$-orthonormal bases for $\mathcal{U}$ and $\mathcal{V}$, respectively, then

$$
\theta_{i}(\mathcal{U}, \mathcal{V})=\arccos \left(\sigma_{i}\right)
$$

where $\sigma_{i}$ is the ith singular value of $U^{*} A V$.

If $\operatorname{dim} \mathcal{U}=\operatorname{dim} \mathcal{V}$, then it is known (see e.g., [12, p. 249]) that the largest principal angle is given by

$$
\theta_{\max }(\mathcal{U}, \mathcal{V})=\max _{u \in S(\mathcal{U})} \min _{v \in S(\mathcal{V})} \measuredangle(u, v)
$$

See [13] for a proof.

We note that $\mathcal{U}$ and $\mathcal{V}$ are orthogonal if and only if $\theta_{1}(\mathcal{U}, \mathcal{V})=\pi / 2$, and that $\mathcal{U}=\mathcal{V}$ if and only if $\theta_{\max }(\mathcal{U}, \mathcal{V})=0$ and $\operatorname{dim} \mathcal{U}=\operatorname{dim} \mathcal{V}$.

In the following lemmas the calligraphic notation $\mathcal{X}(s)$ refers to a subspace which depends on the parameter $s \geq 0$.

Lemma 2.2. Let $\varepsilon \in(0,1)$ and assume that

$$
\lim _{s \rightarrow \infty} \theta_{1}\left(\mathcal{U}_{1}(s), \mathcal{U}_{2}(s)\right)=\pi / 2 .
$$


For sufficiently large $s,\left\|u_{1}+u_{2}\right\|=1$, where $u_{1} \in \mathcal{U}_{1}(s)$ and $u_{2} \in \mathcal{U}_{2}(s)$, implies that

$$
1 /(1+\varepsilon)<\left\|u_{1}\right\|^{2}+\left\|u_{2}\right\|^{2}<1 /(1-\varepsilon) .
$$

Proof. The limit condition of the lemma implies that $\left|\left\langle u_{1}, u_{2}\right\rangle\right|<\left\|u_{1}\right\|\left\|u_{2}\right\| \varepsilon$ for large enough $s$. We have

$$
\left|\left\|u_{1}\right\|^{2}+\left\|u_{2}\right\|^{2}-1\right| \leq 2\left|\left\langle u_{1}, u_{2}\right\rangle\right|<2 \varepsilon\left\|u_{1}\right\|\left\|u_{2}\right\| \leq\left(\left\|u_{1}\right\|^{2}+\left\|u_{2}\right\|^{2}\right) \varepsilon,
$$

from which the lemma follows.

$$
\begin{aligned}
& \text { Lemma 2.3. Let } \mathcal{U}(s)=\operatorname{span}\left\{\mathcal{U}_{1}(s), \mathcal{U}_{2}(s)\right\} \text {. If } \\
& \qquad \lim _{s \rightarrow \infty} \theta_{1}\left(\mathcal{U}_{1}(s), \mathcal{U}_{2}(s)\right)=\lim _{s \rightarrow \infty} \theta_{1}\left(\mathcal{U}_{1}(s), \mathcal{V}(s)\right)=\lim _{s \rightarrow \infty} \theta_{1}\left(\mathcal{U}_{2}(s), \mathcal{V}(s)\right)=\pi / 2,
\end{aligned}
$$

then

$$
\lim _{s \rightarrow \infty} \theta_{1}(\mathcal{U}(s), \mathcal{V}(s))=\pi / 2 .
$$

Proof. Let $u \in \mathcal{U}(s)$ and $v \in \mathcal{V}(s)$ be any vectors such that $\|u\|=\|v\|=1$. We have $u=u_{1}+u_{2}$ where $u_{1} \in \mathcal{U}_{1}(s)$ and $u_{2} \in \mathcal{U}_{2}(s)$ and

$$
|\langle u, v\rangle|=\left|\left\langle u_{1}, v\right\rangle+\left\langle u_{2}, v\right\rangle\right| \leq\left|\left\langle u_{1}, v\right\rangle\right|+\left|\left\langle u_{2}, v\right\rangle\right| .
$$

By Lemma 2.2 the norms of $u_{1}$ and $u_{2}$ are bounded when $s$ is sufficiently large. Hence the right hand side of (2.2) can be forced to be arbitrarily small by taking $s$ large enough.

Proposition 2.4. Suppose $\mathcal{V}(s) \subseteq \operatorname{span}\left\{\mathcal{U}_{1}(s), \mathcal{U}_{2}(s) \ldots, \mathcal{U}_{p}(s)\right\}$ and $\operatorname{dim} \mathcal{U}_{k}(s)=$ $\operatorname{dim} \mathcal{V}(s)$ for a fixed $k \in\{1,2, \ldots, p\}$. If for any $i \neq k$ and any $j \neq \ell$, it holds that

$$
\lim _{s \rightarrow \infty} \theta_{1}\left(\mathcal{V}(s), \mathcal{U}_{i}(s)\right)=\lim _{s \rightarrow \infty} \theta_{1}\left(\mathcal{U}_{j}(s), \mathcal{U}_{\ell}(s)\right)=\pi / 2,
$$

then

$$
\lim _{s \rightarrow \infty} \theta_{\max }\left(\mathcal{V}(s), \mathcal{U}_{k}(s)\right)=0
$$

Proof. Let $\mathcal{W}(s)=\operatorname{span}\left\{\mathcal{U}_{i}(s): i \neq k\right\}$. Lemma 2.3 implies

$$
\lim _{s \rightarrow \infty} \theta_{1}(\mathcal{V}(s), \mathcal{W}(s))=\lim _{s \rightarrow \infty} \theta_{1}\left(\mathcal{U}_{k}(s), \mathcal{W}(s)\right)=\pi / 2 .
$$

Pick $N$ and $\varepsilon \in(0,1)$ such that for any $s>N$ it holds that

$$
\max _{\substack{u_{k} \in \mathcal{U}_{k}(s) \\ w \in \mathcal{W}(s)}} \frac{\left|\left\langle u_{k}, w\right\rangle\right|}{\left\|u_{k}|\|\mid\| w \|\right.}<\varepsilon / 4
$$

and

$$
\max _{\substack{v \in S(\mathcal{V}(s)) \\ w \in S(\mathcal{W}(s))}}|\langle v, w\rangle|<\varepsilon / 2 .
$$


Let $v \in S(\mathcal{V}(s))$ and write $v=u_{k}+w$, where $u_{k} \in \mathcal{U}_{k}(s)$ and $w \in \mathcal{W}(s)$. Due to Lemma 2.2 we may, by possibly choosing a larger $N$, assume that $\left\|u_{k}\right\|<2$ (for any choice of $v$ ), so (2.3) yields $\left|\left\langle u_{k}, w\right\rangle\right| /\|w\|<\varepsilon / 2$. We get

$$
\left|\frac{\left|\left\langle u_{k}, w\right\rangle\right|}{\|w\|}-\|w\|\right| \leq \max _{\widetilde{w} \in S(\mathcal{W}(s))}\left|\left\langle u_{k}, \widetilde{w}\right\rangle+\langle w, \widetilde{w}\rangle\right|=\max _{\widetilde{w} \in S(\mathcal{W}(s))}|\langle v, \widetilde{w}\rangle|<\varepsilon / 2,
$$

where (2.4) is used for the last inequality, and hence $\|w\|<\varepsilon$. Further,

$$
\left\|u_{k}\right\|^{2} \geq\|v\|-\|w\|^{2}-2\left|\left\langle u_{k}, w\right\rangle\right|=1-\|w\|^{2}-2\|w\| \frac{\left|\left\langle u_{k}, w\right\rangle\right|}{\|w\|}>1-2 \varepsilon^{2} .
$$

Note that this holds for any choice of $v=u_{k}+w \in \mathcal{V}(s)$ for $s>N$. Now, by (2.1), we have $\theta_{\max }\left(\mathcal{V}(s), \mathcal{U}_{k}(s)\right)=\arccos (x)$, where

$$
\begin{aligned}
x & =\min _{v \in S(\mathcal{V}(s))} \max _{\widetilde{u}_{k} \in S\left(\mathcal{U}_{k}(s)\right)}\left|\left\langle v, \widetilde{u}_{k}\right\rangle\right| \geq \min _{\substack{u_{k}+w \in S(\mathcal{V}(s)) \\
u_{k} \in \mathcal{U} k(s) \\
w \in \mathcal{W}(s)}}\left|\left\|u_{k}\right\|+\frac{\left\langle w, u_{k}\right\rangle}{\left\|u_{k}\right\|}\right| \\
& \geq \min _{\substack{u_{k}+w \in S(\mathcal{V}(s)) \\
u_{k} \in \mathcal{U}_{k}(s) \\
w \in \mathcal{W}(s)}}\left\|u_{k}\right\|-\|w\|>\sqrt{1-2 \varepsilon^{2}}-\varepsilon .
\end{aligned}
$$

Since $\varepsilon$ can be chosen to be arbitrarily small, the lemma follows.

Proposition 2.5. Suppose the inner product $\langle\cdot, \cdot\rangle$ corresponds to a real symmetric positive definite matrix $A$, so $\langle x, y\rangle=x^{*} A y$ for any vectors $x$ and $y$. If $\operatorname{dim} \mathcal{U}(s)=p$ for $s>N$ and

$$
\lim _{s \rightarrow \infty} \theta_{\max }(\overline{\mathcal{U}(s)}, \mathcal{U}(s))=0,
$$

then for any $\varepsilon>0, \mathcal{U}(s)$ has an $A$-orthonormal basis $\left\{u_{1}, u_{2}, \ldots, u_{p}\right\}$ with $\left\|\operatorname{Im}\left(u_{i}\right)\right\|<$ $\varepsilon, i=1,2, \ldots, p$, for large enough $s$.

Proof. Let $U=\left[u_{1}, u_{2}, \ldots, u_{p}\right]$ be any $A$-orthonormal basis of $\mathcal{U}(s)$ and note that the columns of $\bar{U}$ is an $A$-orthonormal basis of $\overline{\mathcal{U}(s)}$. Since $A$ is real, $U^{T} A U$ is complex symmetric and hence enjoys a singular value decomposition on the form $Q \Sigma Q^{T}$ (also known as a Takagi factorization) [4, Corollary 4.4.4]. By the limit assumption and Theorem 2.1, all singular values are in $(1-\varepsilon, 1]$ for large enough $s$. Define $Y=$ $\left[y_{1}, y_{2}, \ldots, y_{p}\right]=U \bar{Q}$, and note that

$$
Y^{T} A Y=Q^{*} U^{T} A U \bar{Q}=Q^{*} Q \Sigma Q^{T} \bar{Q}=\Sigma .
$$

The columns of $Y$ form an $A$-orthonormal basis of $\mathcal{U}(s)$, and

$$
2\left\|\operatorname{Im}\left(y_{i}\right)\right\|^{2}=\operatorname{Re}\left(y_{i}^{*} A y_{i}-y_{i}^{T} A y_{i}\right)<\varepsilon
$$

for $i=1,2, \ldots, p$.

3. Eigenvalues. In this section we study the eigenvalues of $P_{s}(\lambda)$. Let $(\cdot)^{1 / 2}$ denote the principal square root, and introduce $A=M^{-1 / 2} D M^{-1 / 2}$ and $B=$ $M^{-1 / 2} K M^{-1 / 2}$. Clearly, $P_{s}(\lambda)$ is equivalent to $I \lambda^{2}+s A \lambda+B$, so they have the same Jordan structure. We will repetitively make use of the following linearization:

$$
\underbrace{\left[\begin{array}{cc}
s B^{-1 / 2} A & B^{-1 / 2} \\
-I
\end{array}\right]}_{S} \underbrace{\left[\begin{array}{cc}
-B & -s A
\end{array}\right]}_{\begin{array}{c}
\text { Companion } \\
\text { linearization }
\end{array}} \underbrace{\left[\begin{array}{ll}
B^{1 / 2} & s A
\end{array}\right]}_{S^{-1}}=\left[\begin{array}{cc}
B^{1 / 2} \\
-B^{1 / 2} & -s A
\end{array}\right] .
$$




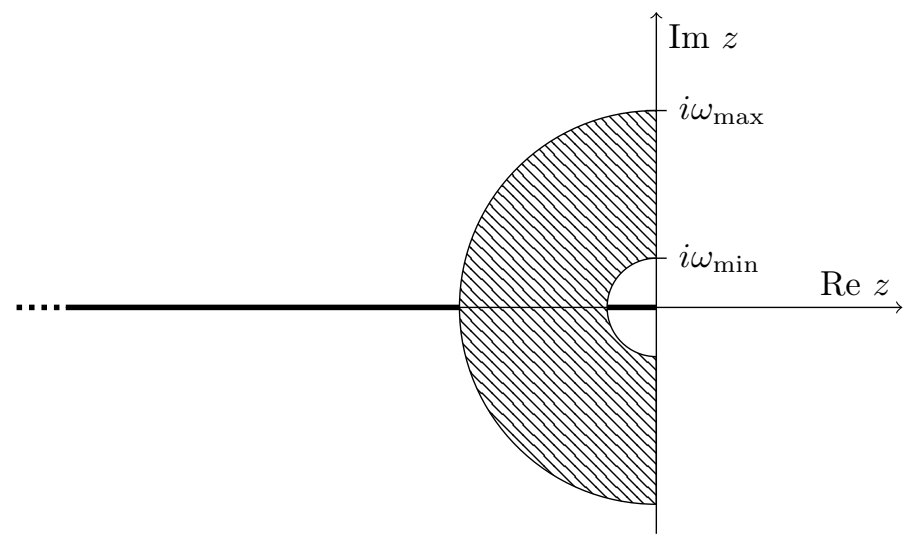

FIG. 3.1. The shaded area is $\mathcal{S}$, and the left and right thick lines are $\mathcal{S}_{\text {out }}$ and $\mathcal{S}_{\text {in }}$ respectively.

Lemma 3.1. If $\lambda_{1}, \lambda_{2}, \ldots, \lambda_{r}$ are the eigenvalues of $s A$, then we have Gerschgorinlike discs

$$
\mathcal{G}_{0}=\left\{z:|z| \leq\|B\|_{2}^{1 / 2}\right\} \quad \text { and } \quad \mathcal{G}_{i}=\left\{z:\left|z-\lambda_{i}\right| \leq\|B\|_{2}^{1 / 2}\right\}
$$

for $i=1,2, \ldots, r$, such that the eigenvalues of $P_{s}(\lambda)$ are contained in the union $\mathcal{G}_{0} \cup \mathcal{G}_{1} \cup \cdots \cup \mathcal{G}_{r}$. Furthermore, $k$ Gerschgorin-like discs contain exactly $k$ eigenvalues (counting multiplicities) if they are disjoint from the remaining discs.

Proof. Apply a real orthogonal similarity transformation to $I \lambda^{2}+A \lambda+B$, to obtain $I \lambda^{2}+\widetilde{A} \lambda+\widetilde{B}$, where $\widetilde{A}$ is diagonal. Note that all but $r$ of the diagonal entries of $\widetilde{A}$ must be zero. Consider the linearization (3.1), with $\widetilde{A}$ and $\widetilde{B}$ in place of $A$ and $B$, respectively. Since $\widetilde{A}$ is normal and $\|\widetilde{B}\|_{2}=\|B\|_{2}$ the lemma follows from $[11$, Theorem 2.1 and Corollary 2.5].

Let $\omega_{\max }=\|B\|_{2}^{1 / 2}$ and $\omega_{\min }=\sigma_{\min }(B)^{1 / 2}$ (where $\sigma_{\min }$ refers to the smallest singular value) and define the following sets:

$$
\mathcal{S}_{\text {in }}=\left\{z:-\omega_{\min }<z<0\right\}, \quad \mathcal{S}_{\text {out }}=\left\{z: z<-\omega_{\max }\right\}
$$

and

$$
\mathcal{S}=\left\{z: \omega_{\min } \leq|z| \leq \omega_{\max }, \operatorname{Re}(z) \leq 0\right\}
$$

See Figure 3.1 for an illustration. Lancaster showed that all non-real eigenvalues of $P_{s}(\lambda)$ lie in the half annulus $\mathcal{S}$ [8, Chapter 9]. Hence any eigenvalues that is not in $\mathcal{S}$ must be in either $\mathcal{S}_{\text {in }}$ or $\mathcal{S}_{\text {out }}$. Our first goal in this section is to bound the number of such eigenvalues. To do so, we need the following lemma.

Lemma 3.2. All eigenvalues of $P_{s}(\lambda)$ that lie in $\mathcal{S}_{\text {in }}$ or $\mathcal{S}_{\text {out }}$ are semi-simple.

Proof. Let $\lambda$ be a real defective eigenvalue of $I \lambda^{2}+s A \lambda+B$, and note that any corresponding real right eigenvector is also a left eigenvector. By [8, Theorem 4.6] there exists an eigenpair $(\lambda, v)$, where $\|v\|_{2}=1$, such that

$$
v^{T}\left(\frac{d}{d \lambda}\left(I \lambda^{2}+s A \lambda+B\right)\right) v=v^{T}(2 I \lambda+s A) v=0 .
$$

This result follows from an arbitrary Jordan decomposition of a certain real linearization. By choosing a Jordan decomposition such that all eigenvectors corresponding 


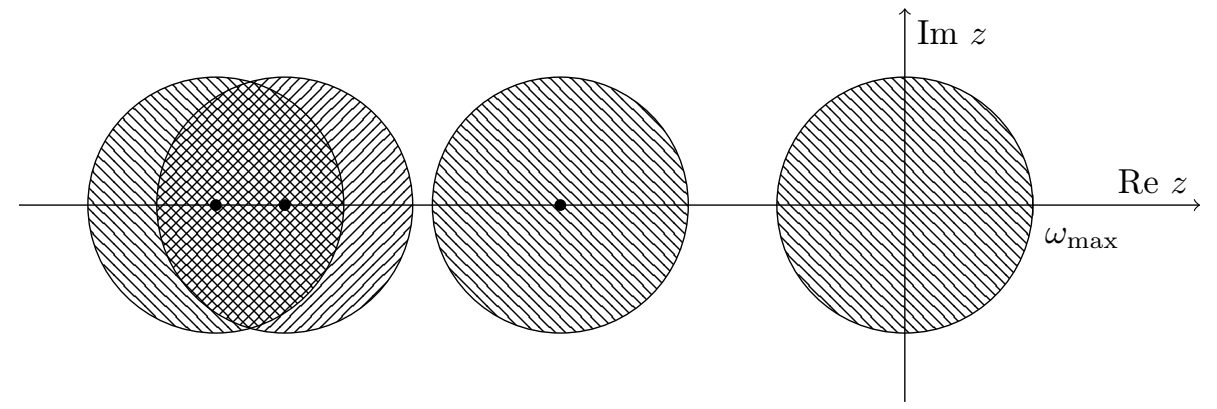

FIG. 3.2. An illustration of what the Gerschgorin-like discs may look like for $r=3$ and large enough s. The disc (from left to right) are $\mathcal{G}_{1}, \mathcal{G}_{2}, \mathcal{G}_{3}, \mathcal{G}_{0}$ and the dots are the nonzero eigenvalues of $-s A$. Lemma 3.1 implies that $\mathcal{G}_{1} \cup \mathcal{G}_{2} \cup \mathcal{G}_{3}$ contains three eigenvalues of $P_{s}(\lambda)$, counting multiplicities.

to $\lambda$ are real, we may assume that $v$ is real as well. If we define $a=s v^{T} A v$ and $b=v^{T} B v$, we have $\lambda=-a / 2$. Further,

$$
v^{T}\left(I \lambda^{2}+s A \lambda+B\right) v=\lambda^{2}+a \lambda+b=\frac{a^{2}}{4}+\frac{a^{2}}{2}+b=0,
$$

which implies $a=2 \sqrt{b}$, and hence $\lambda=-\sqrt{b} \in \mathcal{S}$.

THEOREM 3.3. The sets $\mathcal{S}_{\text {in }}$ and $\mathcal{S}_{\text {out }}$ each contains at most $r$ eigenvalues of $P_{s}(\lambda)$ (counting multiplicities). Furthermore, $r$ eigenvalues go to $-\infty$, and $r$ eigenvalues go to 0 , as $s \rightarrow \infty$.

Proof. By Lemma 3.1, $r$ eigenvalues of $P_{s}(\lambda)$ approach $-\infty$ as $s \rightarrow \infty$, and all remaining eigenvalues lie in $\mathcal{S} \cup \mathcal{S}_{\text {in }}$; see Figure 3.2 for an illustration when $r=3$. We now show that $\mathcal{S}_{\text {out }}$ cannot contain more than $r$ eigenvalues for intermediate values of $s$. Let $L(s)$ denote the linearization (3.1). By Lemma 3.2 all eigenvalues in $\mathcal{S}_{\text {out }}$ are semi-simple and hence differentiable with respect to $s$ [7, Theorem 6]. Consider an eigenvalue $\lambda \in \mathcal{S}_{\text {out }}$ for an arbitrary $s$. Since $\lambda$ is real, the corresponding eigenspace of $L(s)$ has a real basis. If the columns of $W$ is such a basis, it is easy to see that $W=\left[V^{T} B^{1 / 2}, \lambda V^{T}\right]^{T}$, where the columns of $V$ are real eigenvectors with respect to $\lambda$ of the corresponding quadratic matrix polynomial. Furthermore, the columns of $\left[-V^{T} B^{1 / 2}, \lambda V^{T}\right]^{T}$ is a basis of the corresponding left eigenspace, and

$$
\left[\begin{array}{ll}
-V^{T} B^{1 / 2} & \lambda V^{T}
\end{array}\right]\left[\begin{array}{c}
B^{1 / 2} V \\
\lambda V
\end{array}\right]=\lambda^{2} V^{T} V-V^{T} B V
$$

is positive definite since $\lambda^{2}>\omega_{\max }^{2}=\|B\|_{2}$. Suppose, without loss of generality, that $V$ satisfies

$$
\lambda^{2} V^{T} V-V^{T} B V=I,
$$

(otherwise we can replace $V$ by $V Z$ for an appropriate $Z$ ). Then, the derivatives of the eigenvalues that equal $\lambda$ for the considered value of $s$, is given by the eigenvalues of the following matrix [7, Theorem 7],

$$
\left[\begin{array}{ll}
-V^{T} B^{1 / 2} & \lambda V^{T}
\end{array}\right] \frac{\mathrm{d}}{\mathrm{d} s} L(s)\left[\begin{array}{c}
B^{1 / 2} V \\
\lambda V
\end{array}\right]=-\lambda^{2} V^{T} A V,
$$

which is negative semi-definite. Hence all eigenvalues that enter $\mathcal{S}_{\text {out }}$ will stay in $\mathcal{S}_{\text {out }}$ as $s \rightarrow \infty$. 
Let $\sim$ denote the equivalence relation for matrix polynomials. To compute the number of eigenvalues in $\mathcal{S}_{i n}$, we consider

$$
\operatorname{rev} P_{s}(\lambda)=K \lambda^{2}+s D \lambda+M \sim I \lambda^{2}+s \underbrace{K^{-1 / 2} D K^{-1 / 2}}_{\widehat{A}} \lambda+\underbrace{K^{-1 / 2} M K^{-1 / 2}}_{\widehat{B}},
$$

and note that $\widehat{B}$ is similar to $B^{-1}$. It is easy to see that $\lambda$ is an eigenvalue of $P_{s}(\lambda)$ if and only if $1 / \lambda$ is an eigenvalue of $\operatorname{rev} P_{s}(\lambda)$ with the same algebraic multiplicity. The proved part of the theorem implies that $r$ eigenvalues of rev $P_{s}(\lambda)$ go to $-\infty$ as $s \rightarrow \infty$, so $r$ eigenvalues of $P_{s}(\lambda)$ must go to zero (along the negative real axis). Furthermore, rev $P_{s}(\lambda)$ has at most $r$ eigenvalues in $\left\{z: z<-\|\widehat{B}\|_{2}^{1 / 2}\right\}$ so $P_{s}(\lambda)$ has at most $r$ eigenvalues in

$$
\left\{z:-\|\widehat{B}\|_{2}^{-1 / 2}<z<0\right\}=\left\{z:-\sigma_{\min }(B)^{1 / 2}<z<0\right\}=\mathcal{S}_{\text {in }} .
$$

We classify the eigenvalues of $P_{s}(\lambda)$ based on whether or not they depend on $s$. Eigenvalues are said to be affected (by damping) if they depend on $s$, and unaffected otherwise. To be more precise, let the columns of $V=\left[V_{1}, V_{2}\right]$ be a real $M$-orthogonal basis where range $\left(V_{1}\right)$ is spanned by all eigenvectors of $P_{0}(\lambda)$ that are in the null space of $D$. If we apply the congruence transformation defined by $V$ to $P_{s}(\lambda)$, the resulting matrix polynomial decomposes into the direct sum of an "undamped part" and a "damped part":

$$
V^{T} P_{s}(\lambda) V=\left(I_{k} \lambda^{2}+K_{1}\right) \oplus\left(I_{n-k} \lambda^{2}+s D_{2} \lambda+K_{2}\right) .
$$

Here $k=\operatorname{rank}\left(V_{1}\right), V_{1}^{T} K V_{1}=K_{1}, V_{2}^{T} K V_{2}=K_{2}$ and $V_{2}^{T} D V_{2}=D_{2}$. The eigenvalues of $I_{k} \lambda^{2}+K_{1}$ are clearly independent of $s$ and hence unaffected. In particular they must be purely imaginary. The next lemma shows that the eigenvalues of $I_{n-k} \lambda^{2}+$ $s D_{2} \lambda+K_{2}$ are the affected eigenvalues, and furthermore that they are only purely imaginary for $s=0$.

Lemma 3.4. If $\lambda$ is an eigenvalue of $I_{n-k} \lambda^{2}+s D_{2} \lambda+K_{2}$ for some $s>0$, then $\operatorname{Re}(\lambda)<0$.

Proof. Assume the contrary and let $(\lambda=\omega i, x)$ be an eigenpair such that $\omega \in \mathbb{R} \backslash\{0\}$. If $v=V_{2} x$, then

$$
v^{*}\left(K-\omega^{2} M+i \omega s D\right) v=0,
$$

and since $v^{*} M v, v^{*} D v$ and $v^{*} K v$ are real, we must have $v^{*} D v=0$. Because $D$ is symmetric positive semi-definite, this implies that $D v=0$. But then $(\lambda, v)$ is an eigenvalue of $P_{0}(\lambda)$ for which $D v=0$. This contradicts that $v \in \operatorname{range}\left(V_{2}\right)$.

As $s \rightarrow \infty$ we know from Theorem 3.3 that $r$ eigenvalues enter $\mathcal{S}_{\text {out }}$ and go to $-\infty$, and $r$ other eigenvalues enter $\mathcal{S}_{\text {in }}$ and go 0 . We now focus on the remaining affected eigenvalues, that is, the ones that stay in $\mathcal{S}$ as $s \rightarrow \infty$.

THEOREM 3.5. For large enough $s$, the affected eigenvalues in $\mathcal{S}$ are continuous functions of $s$ that converge to purely imaginary points.

Proof. Recall that $D=R R^{T}$ with $R \in \mathbb{R}^{n \times r}$, and define $t=1 / s, p(\lambda)=$ $\operatorname{det}\left(M \lambda^{2}+K\right), Q(\lambda)=\lambda R^{T}\left(M \lambda^{2}+K\right)^{-1} R$ and

$$
q_{t}(\lambda)=\operatorname{det}\left(t p(\lambda) I_{r}+p(\lambda) Q(\lambda)\right)
$$


By Lemma 3.4, no affected eigenvalues are roots of $p(\lambda)$ for $s>0$. Thus, for $t>0$, any root $\lambda_{i}$ of $q_{t}(\lambda)$ that is not a root of $p(\lambda)$ is an affected eigenvalue. To see this, we simply note that

$$
0=q_{t}\left(\lambda_{i}\right)=p\left(\lambda_{i}\right)^{r} \operatorname{det}\left(t I_{r}+Q\left(\lambda_{i}\right)\right)=p\left(\lambda_{i}\right) \operatorname{det}\left(t I_{r}+Q\left(\lambda_{i}\right)\right)=\operatorname{det} P_{s}\left(\lambda_{i}\right),
$$

where the matrix determinant lemma (related to the Sherman-Morrison formula) has been used for the last equality. On noting that $\left(M \lambda^{2}+K\right)^{-1}=\operatorname{Adj}\left(M \lambda^{2}+K\right) / p(\lambda)$, it is easy to see that $p(\lambda) Q(\lambda)$ is a matrix polynomial, so $q_{t}(\lambda)$ is a polynomial, and further

$$
\operatorname{deg} q_{t}(\lambda)= \begin{cases}2 n r & \text { if } t \neq 0, \\ 2 n r-r & \text { if } t=0 .\end{cases}
$$

From the context, it is natural to consider $q_{0}(\lambda)$ as a polynomial of grade $2 n r$ (see [3]), in which case we $q_{0}(\lambda)$ has $r$ infinite roots. Consider a finite root $\lambda_{i}$ of $q_{0}(\lambda)$ of multiplicity $\alpha$. In a neighborhood of $t=0$, the solutions $\lambda$ of $q_{t}(\lambda)=0$ can be expanded in Puiseux series in $t$, and $\alpha$ of these series (counting multiplicities) equal $\lambda_{i}$ at $t=0$ [6, Chapter 5]. Thus, $\alpha$ roots of $q_{t}(\lambda)$, seen as functions of $t$, converge to $\lambda_{i}$ as $t \rightarrow 0$. This shows that $2 n r-r$ roots of $q_{t}(\lambda)$ converge to the finite roots of $q_{0}(\lambda)$ as $t \rightarrow 0$. Furthermore, from Theorem 3.3 we know that the remaining $r$ roots go to $-\infty$ as $t \rightarrow 0$.

To show that the finite roots of $q_{0}(\lambda)$ are purely imaginary we note that they are all eigenvalues of $p(\lambda) Q(\lambda)$. Since $p(-\lambda) Q(-\lambda)=-p(\lambda) Q(\lambda)^{T}, p(\lambda) Q(\lambda)$ is T-odd, so $\lambda_{i}$ is an eigenvalue if and only if $-\lambda_{i}$ also is an eigenvalue [10, Theorem 4.2]. Therefore, if $q_{0}(\lambda)$ has a root with negative real part, it also has a root with positive real part. But this is impossible. Indeed, $q_{t}(\lambda)$ has a root with positive real part for some $t>0$ only if there is an affected eigenvalue with positive real part, a contradiction.

Let $L(s)$ denote the linearization (3.1). The matrix $L(s)$, and hence the matrix polynomial $P_{s}(\lambda)$, has a constant number, $k$, of distinct eigenvalues for all but a finite number of values of $s$, known as exceptional points [5, p. 64]. Exceptional points are in general non-real, so for the sake of the argument, we temporarily expand the scope and allow non-real values of $s$. Now, in any simple domain not containing any exceptional points, we have a Jordan decomposition (albeit not in its usual likeness)

$$
L(s)=\sum_{i=1}^{k} P_{i}(s) \lambda_{i}(s)+D_{i}(s),
$$

where $P_{i}(s), \lambda_{i}(s)$ and $D_{i}(s)$ denote the eigenprojections, (distinct) eigenvalues and eigennilpotents, respectively, and all are analytic in the considered domain [5, p. 68]. The following observation was made in [9, Theorem 3.3]: for purely imaginary $s, L(s)$ is skew-Hermitian so the eigennilpotents must vanish. Because any simple domain, free of exceptional points, can be expanded to contain an interval of the imaginary axis, in a manner that avoids including exceptional points, the eigennilpotents must vanish identically for any non-exceptional $s$. Put simply, defective eigenvalues can only exist for a finite number of exceptional values of $s$. This implies the following result.

TheOrem 3.6. For large enough s, all eigenvalues of $P_{s}(\lambda)$ are semi-simple.

4. Eigenspaces. Let $\langle\cdot, \cdot\rangle$ be the $M$-inner product and $\|\cdot\|$ the induced norm. In the undamped case, $s=0$, the eigenspaces of $P_{s}(\lambda)$ corresponding to complex conjugate eigenvalues are identical, and any other two eigenspaces are orthogonal with 
respect to $\langle\cdot, \cdot\rangle$. Furthermore, all eigenspaces have real bases. The same is, in general, not true when $s>0$. Our goal in this section is to show that for large enough $s$, the same properties are almost true if we restrict ourselves to the eigenspaces corresponding to eigenvalues in $\mathcal{S}$ and group together eigenspaces corresponding to "close" eigenvalues; more precise statements will be made in Theorem 4.1 and Corollary 4.2.

Suppose $N$ is big enough so there are no exceptional points in $(N, \infty)$; such $N$ exists due to Theorem 3.6. Then there is a constant $k$ such that $P_{s}(\lambda)$ has exactly $k$ distinct eigenvalues $\lambda_{1}(s), \lambda_{2}(s), \ldots, \lambda_{k}(s)$ for $s>N$, which are analytic functions of $s$. Let $\mathcal{V}_{i}(s)$ denote the eigenspace corresponding to $\lambda_{i}(s)$ and define

$$
\mathcal{U}_{z}(s)=\operatorname{span}\left\{\mathcal{V}_{i}(s): \lim _{s \rightarrow \infty} \lambda_{i}(s)=z\right\},
$$

for $s>N$. We shall prove the following result.

TheOREM 4.1. Let $z_{1}, z_{2}, \ldots, z_{2 p}$ denote the distinct nonzero points on the imaginary axis to which some eigenvalue of $P_{s}(\lambda)$ converges as $s \rightarrow \infty$. If $\theta_{1}(\cdot, \cdot)$ and $\theta_{\max }(\cdot, \cdot)$ refer to the smallest and largest principal angles with respect to $\langle\cdot, \cdot\rangle$, respectively, then the following hold:

(a) If $z_{i} \neq \bar{z}_{j}$ and $z_{i} \neq z_{j}$ then $\lim _{s \rightarrow \infty} \theta_{1}\left(\mathcal{U}_{z_{i}}(s), \mathcal{U}_{z_{j}}(s)\right)=\pi / 2$.

(b) $\lim _{s \rightarrow \infty} \theta_{1}\left(\mathcal{U}_{z_{i}}(s), \mathcal{U}_{-\infty}(s)\right)=\pi / 2$.

(c) $\lim _{s \rightarrow \infty} \theta_{1}\left(\mathcal{U}_{z_{i}}(s), \mathcal{U}_{0}(s)\right)=\pi / 2$.

(d) $\lim _{s \rightarrow \infty} \theta_{\max }\left(\mathcal{U}_{z_{i}}(s), \mathcal{U}_{\bar{z}_{i}}(s)\right)=0$.

(e) $\lim _{s \rightarrow \infty} \theta_{\max }\left(\mathcal{U}_{-\infty}(s), \mathcal{U}_{0}(s)\right)=0$.

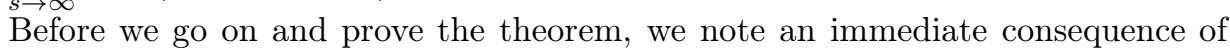
part (d).

COROLlaRY 4.2. For any $\varepsilon>0, \mathcal{U}_{z_{i}}(s)$ has an $M$-orthonormal basis $\left\{u_{1}, u_{2}, \ldots, u_{k}\right\}$ where $\left\|\operatorname{Im}\left(u_{j}\right)\right\|<\varepsilon, j=1,2, \ldots, k$, for large enough $s$.

Proof. The corollary follows immediately from Proposition 2.5.

To prove Theorem 4.1, we need the following lemma.

LEMma 4.3. Let $\mathcal{S}$ be defined by (3.3) and consider eigenpairs $\left(\lambda_{i}, v_{i}\right)$ and $\left(\lambda_{j}, v_{j}\right)$ of $P_{s}(\lambda)$, for some $s>0$, for which $\left\|v_{i}\right\|=\left\|v_{j}\right\|=1$ and $\lambda_{i} \in \mathcal{S}$. There are constants $c_{1}$ and $c_{2}$ which are independent of $v_{i}, v_{j}$ and $s$, such that the following bounds hold:

(a) If $\lambda_{j} \in \mathcal{S}$ then $\left|v_{i}^{*} D v_{j}\right| \leq\left(c_{1} / s\right)^{2}$.

(b) $\left|v_{i}^{*} D v_{j}\right| \leq c_{2} / s$.

Proof. Recall that $D=R R^{T}$ with $R \in \mathbb{R}^{n \times r}$. We have

$$
R R^{T} v_{i}=-\left(\lambda_{i} s\right)^{-1}\left(M \lambda_{i}^{2}+K\right) v_{i} .
$$

Left multiplication with $M^{-1 / 2}$ times the Moore-Penrose pseudo-inverse $R^{\dagger}$ and taking norms yields

$$
\left\|M^{-1 / 2} R^{T} v_{i}\right\|=\frac{\left\|M^{-1 / 2} R^{\dagger}\left(M \lambda_{i}^{2}+K\right) v_{i}\right\|}{\left|\lambda_{i} s\right|} .
$$

Since $\left|\lambda_{i}\right|$ is bounded from below and above there is a constant $c_{1}$ such that $\left\|M^{-1 / 2} R^{T} v_{i}\right\| \leq c_{1} / s$, for any $s$ and any choice of $v_{i}$. For case (a), an analogous argument gives $\left\|M^{-1 / 2} R^{T} v_{j}\right\| \leq c_{1} / s$ and Cauchy-Schwartz inequality yields

$$
\begin{aligned}
\left|v_{i}^{*} D v_{j}\right| & =\left|\left\langle M^{-1 / 2} R^{T} v_{i}, M^{-1 / 2} R^{T} v_{j}\right\rangle\right| \\
& \leq\left\|M^{-1 / 2} R^{T} v_{i}\right\|\left\|M^{-1 / 2} R^{T} v_{j}\right\| \\
& \leq\left(c_{1} / s\right)^{2} .
\end{aligned}
$$


Similarly, for part (b), we have

$$
\left|v_{i}^{*} D v_{j}\right| \leq\left\|M^{-1 / 2} R^{T} v_{i}\right\|\left\|M^{-1 / 2} R^{T} v_{j}\right\| \leq c_{2} / s .
$$

for $c_{2}=\left\|M^{-1 / 2} R^{T}\right\| c_{1}$.

Proof of Theorem 4.1 For $i=1,2, \ldots, 2 p$, define $\mathcal{B}_{i}=\left\{z:\left|z-z_{i}\right| \leq \delta\right\}$ where $\delta>0$ is small enough so $\mathcal{B}_{i} \cap \mathcal{B}_{j}=\emptyset$ for $i \neq j$, and let

$$
\gamma=\min _{i \neq j} \operatorname{dist}\left(\mathcal{B}_{i}, \mathcal{B}_{j}\right)
$$

Choose $N>0$ such that for $s>N$ it holds that $\mathcal{B}_{i}$ contains all eigenvalues of $P_{s}(\lambda)$ that converge to $z_{i}$. The $\mathcal{B}_{i}$ s will hereafter be referred to as "limit balls."

Now, pick $\varepsilon>0$ and let $c_{1}$ and $c_{2}$ be the constants from Lemma 4.3. By possibly choosing an even larger $N$, we may assume that $N>c_{1}^{2} /(\varepsilon \gamma)$ and that all eigenvalues in $\mathcal{S}_{\text {out }}$, defined in (3.2), have modulus greater than $c_{2} / \varepsilon$. Consider two eigenpairs $\left(\lambda_{i}, v_{i}\right)$ and $\left(\lambda_{j}, v_{j}\right)$ for which $\left\|v_{i}\right\|=\left\|v_{j}\right\|=1$. If $\lambda_{i}$ and $\lambda_{j}$ belong to different limit balls that are not complex conjugate sets, then we have for any real $s$ that

$$
\begin{aligned}
\bar{\lambda}_{i}^{2} v_{i}^{*} M v_{j} & =\left(\lambda_{i}^{2} M v_{i}\right)^{*} v_{j} \\
& =\left(-\left(s D \lambda_{i}+K\right) v_{i}\right)^{*} v_{j} \\
& =v_{i}^{*}\left(-\left(s D \bar{\lambda}_{i}+K\right)\right) v_{j} \\
& =v_{i}^{*}\left(s D\left(\lambda_{j}-\bar{\lambda}_{i}\right)-\left(s D \lambda_{j}+K\right)\right) v_{j} \\
& =v_{i}^{*}\left(s D\left(\lambda_{j}-\bar{\lambda}_{i}\right) v_{j}-\left(s D \lambda_{j}+K\right) v_{j}\right) \\
& =s\left(\lambda_{j}-\bar{\lambda}_{i}\right) v_{i}^{*} D v_{j}+\lambda_{j}^{2} v_{i}^{*} M v_{j}
\end{aligned}
$$

and further

$$
-v_{i}^{*} M v_{j}=\frac{s v_{i}^{*} D v_{j}}{\bar{\lambda}_{i}+\lambda_{j}}
$$

From (4.1) we have $\left|\bar{\lambda}_{i}+\lambda_{j}\right| \geq \gamma$, and by part (a) of Lemma $4.3\left|s v_{i}^{*} D v_{j}\right| \leq c_{1}^{2} / s$. Thus, $\left|v_{i}^{*} M v_{j}\right|<\varepsilon$ for $s>N$. Since this bound is independent of which normalized eigenvectors $v_{i}$ and $v_{j}$ we picked, and $\varepsilon>0$ is arbitrary, we have proved part (a) of the theorem.

If $\lambda_{i}$ is in a limit ball and $\lambda_{j} \in \mathcal{S}_{\text {out }}$, then $v_{i}$ and $v_{j}$ also satisfy (4.2). By part (b) of Lemma $4.3\left|s v_{i}^{*} D v_{j}\right| \leq c_{2}$ and

$$
\left|\bar{\lambda}_{i}+\lambda_{j}\right| \geq\left|\lambda_{j}\right|>c_{2} / \varepsilon
$$

Hence, $\left|v_{i}^{*} M v_{j}\right|<\varepsilon$ for $s>N$, and we have concluded part (b) of the theorem.

Since rev $P_{s}(\lambda)$ has the same eigenspaces as $P_{s}(\lambda)$, part (c) follows immediately if we consider part (b) for the reversed matrix polynomial.

For $s>N$, the eigenvectors corresponding to the eigenvalues of $P_{s}(\lambda)$ in $\mathcal{B}_{i}$ and $\mathcal{S}_{\text {out }}$, span the subspaces $\mathcal{U}_{z_{i}}(s)$ and $\mathcal{U}_{-\infty}(s)$, respectively. Furthermore, due to Theorem 3.6, $\operatorname{dim} \mathcal{U}_{z_{i}}(s)$ and $\operatorname{dim} \mathcal{U}_{-\infty}(s)$ equals the sums of the algebraic multiplicities of all eigenvalues in $\mathcal{B}_{i}$ and $\mathcal{S}_{\text {out }}$, respectively. Since $z_{1}, z_{2}, \ldots, z_{2 p}$ can be paired into complex conjugates, we may assume, without loss of generality, that $\operatorname{Im}\left(z_{i}\right)>0$ for $i=1,2, \ldots, p$. We have

$$
\operatorname{dim} \mathcal{U}_{z_{1}}+\operatorname{dim} \mathcal{U}_{z_{2}}+\cdots+\operatorname{dim} \mathcal{U}_{z_{p}}+\operatorname{dim} \mathcal{U}_{-\infty}=n
$$


Part (a) and part (b), which we just proved, imply that

$$
\lim _{s \rightarrow \infty} \theta_{1}\left(\mathcal{U}_{\bar{z}_{i}}(s), \mathcal{U}_{z}(s)\right)=\pi / 2
$$

for $z \in\left\{z_{1}, z_{2}, \ldots, z_{p},-\infty\right\} \backslash\left\{z_{i}\right\}$. Hence, part (d) follows from Proposition 2.4. Similarly, we have

$$
\lim _{s \rightarrow \infty} \theta_{1}\left(\mathcal{U}_{0}(s), \mathcal{U}_{z}(s)\right)=\pi / 2
$$

for $z \in\left\{z_{1}, z_{2}, \ldots, z_{p}\right\}$, so also part (e) follows from Proposition 2.4.

Acknowledgements. I thank Lijing Lin and Françoise Tisseur for their comments on the paper.

\section{REFERENCES}

[1] Seismic dampers \& seismic protection products. Retrieved from http://taylordevices.com/dampers-seismic-protection.html Accessed: 2014-0223.

[2] A. Björck and G. H. Golub. Numerical methods for computing angles between linear subspaces. Mathematics of Computation, 27(123):579-594, 1973.

[3] C. M. D. Steven Mackey, Niloufer Mackey and V. Mehrmann. Möbius transformations of matrix polynomials. MIMS EPrint 2014.2, Manchester Institute for Mathematical Sciences, The University of Manchester, UK, January 2014.

[4] R. A. Horn and C. R. Johnson. Matrix Analysis. Cambridge University Press, Cambridge, UK, 1985.

[5] T. Kato. Pertubation Theorey for Linear Operators. Springer-Verlag, Berlin Heidelberg, Germany, 1995. Reprint of the 1980 Edition.

[6] K. Knopp. Theory of Functions. Dover Publications, inc., New York, USA, 1947. English translation, Part II.

[7] P. Lancaster. On eigenvalues of matrices dependent on a parameter. Numerische Mathematik, 6:377-964, 1964 .

[8] P. Lancaster. Lambda-matrices and Vibrating Systems. Dover Publications, inc., New York, USA, 2002.

[9] N. B. Langer, H. and K. Veselić. Perturbation of the eigenvalues of quadratic matrix polynomials. SIAM J. Matrix Anal. Appl., 13(2):474-489, 1992.

[10] D. S. Mackey, N. Mackey, C. Mehl, and V. Mehrmann. Jordan structures of alternating matrix polynomials. Linear Algebra and its Applications, 432(4):867-891, 2010.

[11] D. Meyer and K. Veselić. On some new inclusion theorems for the eigenvalues of partitioned matrices. Numerische Mathematik, 34(4):431-437, 1980.

[12] G. Stewart. Matrix Algorithms, Volume II: Eigensystems. Society for Industrial and Applied Mathematics, Philadelphia, PA, USA, 2001.

[13] L. Taslaman. The principal angles and the gap. MIMS EPrint 2014.9, Manchester Institute for Mathematical Sciences, The University of Manchester, UK, Februari 2014. 